\title{
Second-Phase Defects in Ni-Mn-Ga Ferromagnetic Shape-Memory Alloys
}

\author{
M.L. Richard,* J. Feuchtwanger*, B. Peterson*, S.M. Allen*, R.C. OHandley* \\ * Department of Materials Science and Engineering, Massachusetts Institute of Technology, 77 Mas- \\ sachusetts Ave, Cambridge, MA 02139
}

Field-induced twin-boundary motion is the mechanism responsible for the magnetic shape-memory effect observed in Ni-Mn-Ga based alloys. Through the reorientation of twin variants with an applied magnetic field strains of up to $6 \%$ have be measured in alloys with the tetragonal/5M structure [1] and 10\% strain has been documented in the orthorhombic/14M martensitic structure [2].

Twin-boundary motion has been shown by Marioni et al. [3] to be a stochastic process, suggesting the important role of defects in the motion of boundaries under an applied magnetic field. Marioni has shown that a distribution of defects energies exists in a typical Ni-Mn-Ga single crystal and these defects act as twin-boundary pinning sites. The work presented here documents several types of second-phase defects found in Ni-Mn-Ga single crystals through the use of TEM and STEM microanalysis. The impurities discovered in several of the single-crystal boules indicate the sensitivity of this alloy system to the presence of other components. The relative pinning strengths of the different types of defects were estimated based on the size and volume fraction present. These strengths are related to the observed field-induced twin-boundary motion behavior.

Three types of second-phase particles were identified, each originating from a different source. Large sulfide inclusions were found in varying amounts in several different single-crystal boules (Figure 1a). These particles are from 500-1000 nm in size and are preferentially thinned during sample preparation, making the determination of their exact chemical composition difficult. The sulfur is present due to impurities in all commercially available Mn. Depending on the volume fraction of these sulfide defects, their presence can prevent twin-boundary motion.

In an effort to improve the quality of the crystals the manganese starting material was purified through a sublimation process. However this led to contamination with tantalum due to the use of a Ta substrate for deposition during the purification process. Large Ta inclusions were discovered in crystals grown with the purified material (Figure 1b). These particles were single-crystalline and were surrounded by a large degree of plastic deformation. The tantalum inclusions were not present in sufficient volume fraction in order to prevent twin-boundary motion. However, the presence of the inclusions did lead to premature fracture of single-crystals during actuation. Large numbers of the Ta particles were seen on the fracture surface of several crystals after failure.

Small titanium precipitates were also identified in samples from one single-crystal boule, ranging from 5-20 $\mathrm{nm}$ in size (Figure 2). These particles appear to remain coherent with the matrix as can be seen from the Ashby-Brown [4,5] contrast in Figure 2. The precipitates often appear in large clusters distributed throughout the crystal. The Ti was identified as originating from furnace contamination from the previous growth of a $\mathrm{Nb}$-Ti alloy. Due to their small size, the Ti-particles appear to act as pinning sites that can be overcome with the application of a large enough magnetic field. 


\section{References}

[1] S.J. Murray et al., Appl. Phys. Lett. 77(2000) 886.

[2] P. Mullner, V.A. Chernenko, G. Kostorz., J. Appl. Phys. 93(2004) 1531.

[3] M.A. Marioni, S.M. Allen, R.C. OHandley et al., Appl. Phys. Lett. 84(2005) 4071.

[4] M.F. Ashby, L.M. Brown. Phil. Mag. 8(1963) 1083.

[51 M F Ashhv I. M Rrown Phil Mas 8(1963) 1649

58.

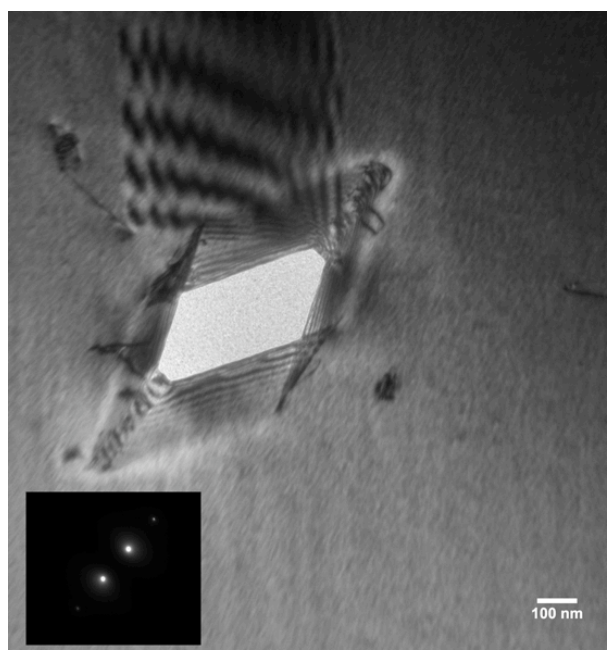

(a)

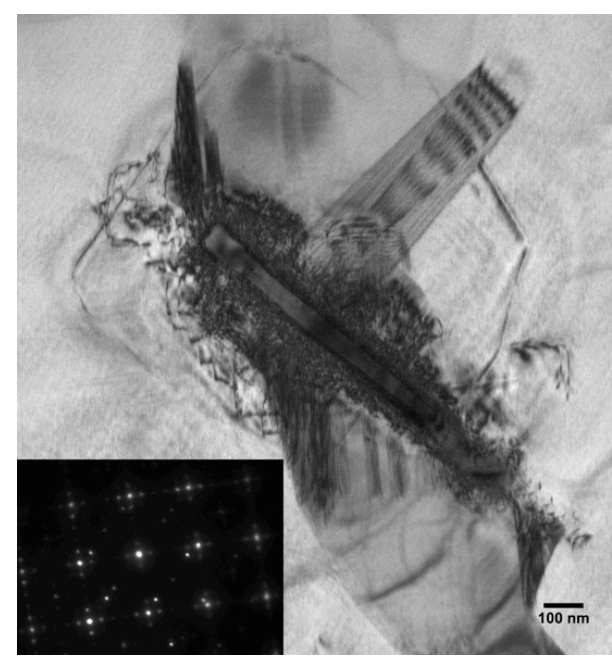

(b)

Figure 1: (a) Bright-field micrograph of a large sulfide inclusion ( $\mathrm{g}=[220])$. (b) Ta inclusion found in a sample taken from a crystal grown with high-purity manganese, $B=[001]$. Scale bars are 100 nm.

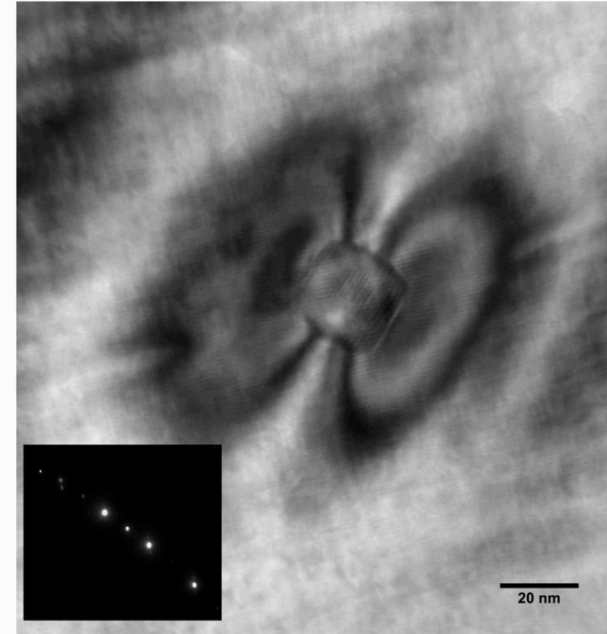

(a)

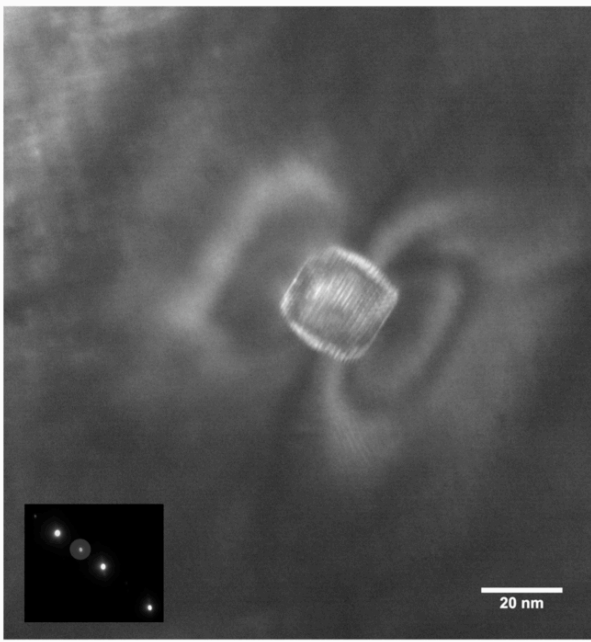

(b)

Figure 2: High magnification (a) bright-field and (b) dark field micrograph of a coherent precipitate found in sample TL8, $\mathrm{g}=[400]$. Scale bar is $20 \mathrm{~nm}$. 